\title{
A CLUNIE LEMMA FOR DIFFERENCE AND $q$-DIFFERENCE POLYNOMIALS
}

\author{
ZHI-BO HUANG and ZONG-XUAN CHEN ${ }^{凶}$
}

(Received 28 November 2008)

\begin{abstract}
The main purpose of this paper is to prove difference and $q$-difference counterparts of the Clunie lemma from the Nevanlinna theory of differential polynomials, where the difference and $q$-difference polynomials can contain many terms of maximal total degree in $f(z)$ and its $(q$-)shifts.
\end{abstract}

2000 Mathematics subject classification: primary 30D35; secondary 39B32.

Keywords and phrases: Clunie lemma, difference polynomial, $q$-difference polynomial.

\section{Introduction}

The original version of the well-known Clunie lemma in Nevanlinna theory can be found in [3] and [6, Lemma 2.4.2 and Proposition 9.2.3]. A slightly more general version of the Clunie lemma can be found in [5, pp. 218-220], and [6, Lemma 2.4.5]. Recently, the additional assumptions in the He-Xiao variant of the Clunie lemma have been removed by Yang and Ye in [8, Theorem 1]. The Clunie lemma has numerous applications in the study of complex differential equations.

We first fix some difference polynomials of the following types:

$$
\begin{aligned}
& P(z, f)=\sum_{\lambda \in I} a_{\lambda}(z) \prod_{j=1}^{\sigma_{\lambda}} f\left(z+\alpha_{\lambda, j}\right)^{l_{\lambda, j},}, l_{\lambda}=\sum_{j=1}^{\sigma_{\lambda}} l_{\lambda, j}, \\
& Q(z, f)=\sum_{\mu \in J} b_{\mu}(z) \prod_{j=1}^{\tau_{\mu}} f\left(z+\beta_{\mu, j}\right)^{m_{\mu, j}},
\end{aligned}
$$

where $I$ and $J$ are index sets, $\alpha_{\lambda, j} \in \mathbb{C}, \beta_{\mu, j} \in \mathbb{C}$, and the coefficients of the difference polynomials (1.1) and (1.2) are small functions as understood in the usual Nevanlinna theory; that is, their characteristic is of type $S(r, f)$. Set

$$
c=\max _{\lambda, j}\left\{\left|\alpha_{\lambda, j}\right|\right\} .
$$

The project was supported by the National Natural Science Foundation of China (No. 10871076), and partly supported by the School of Mathematical Sciences Foundation of SCNU, PR China.

(C) 2009 Australian Mathematical Publishing Association Inc. 0004-9727/2009 \$16.00 
There were no results about difference polynomials until two similar results on a difference analogue of the logarithmic derivative lemma were presented in [2, 4]. Then Halburd and Korhonen [4] applied the lemma to difference equations to obtain a difference counterpart of the Clunie lemma, as follows.

THEOREM A [4]. Let $f(z)$ be a nonconstant meromorphic solution of

$$
f(z)^{n} P(z, f)=Q(z, f),
$$

where $P(z, f)$ and $Q(z, f)$ are difference polynomials of types (1.1) and (1.2) respectively, and let $0<\delta<1$ and $\varepsilon>0$. If the degree of $Q(z, f)$ as a polynomial in $f(z)$ and its shifts is at most $n$, then

$$
m(r, P(z, f))=o\left(\frac{T(r+c, f)^{1+\varepsilon}}{r^{\delta}}\right)+o(T(r, f)),
$$

for all $r$ outside of a possible exceptional set with finite logarithmic measure.

In particular, if the order $\rho(f)$ of $f(z)$ is finite, then

$$
m(r, P(z, f))=o\left(\frac{T(r+c, f)}{r^{\delta}}\right)+o(T(r, f)),
$$

for all $r$ outside of a possible exceptional set with finite logarithmic measure.

Subsequently, Laine and Yang used the difference analogue of the logarithmic derivative lemma proved in [2] and obtained a more general version of a difference counterpart of the Clunie lemma as follows.

THEOREM B [7]. Let $f(z)$ be a transcendental meromorphic solution of finite order $\rho$ of a difference equation of the form

$$
U(z, f) P(z, f)=Q(z, f)
$$

where $U(z, f), P(z, f)$ and $Q(z, f)$ are difference polynomials such that the total degree $\operatorname{deg}_{f} U(z, f)=n$ in $f(z)$ and its shifts, and $\operatorname{deg}_{f} Q(z, f) \leq n$. Moreover, we assume that $U(z, f)$ contains just one term of maximal total degree in $f(z)$ and its shifts. Then for each $\varepsilon>0$,

$$
m(r, P(z, f))=O\left(r^{\rho-1+\varepsilon}\right)+S(r, f),
$$

possibly outside an exceptional set of finite logarithmic measure.

Now consider replacing difference polynomials by $q$-difference polynomials. Here a $q$-difference polynomial of $f(z)$ for $q \in \mathbb{C} \backslash\{0,1\}$ is a polynomial in $f(z)$ and finitely many of its $q$-shifts $f(q z), f\left(q^{2} z\right), \ldots, f\left(q^{n} z\right)$ with meromorphic coefficients in the sense that their Nevanlinna characteristic functions are $o(T(r, f))$ on a set of logarithmic density 1. In [1], Barnet et al. obtained a $q$-difference analogue of the logarithmic derivative lemma and applied it to a $q$-difference equation. Then they proved a $q$-difference counterpart of the Clunie lemma as follows. 
THEOREM C [1]. Let $f(z)$ be a nonconstant zero-order meromorphic solution of

$$
f(z)^{n} P(z, f)=Q(z, f),
$$

where $P(z, f)$ and $Q(z, f)$ are $q$-difference polynomials in $f(z)$ and its $q$-shifts. If the degree of $Q(z, f)$ as a polynomial in $f(z)$ and its $q$-shifts is at most $n$, then

$$
m(r, P(z, f))=o(T(r, f))
$$

on a set of logarithmic density 1.

Subsequently Laine and Yang generalized this and obtained a more general version of a $q$-difference counterpart of the Clunie lemma as follows.

THEOREM D [7]. Let $f(z)$ be a transcendental meromorphic solution of zero order of a $q$-difference equation of the form

$$
U_{q}(z, f) P_{q}(z, f)=Q_{q}(z, f),
$$

where $U_{q}(z, f), P_{q}(z, f)$ and $Q_{q}(z, f)$ are $q$-difference polynomials such that the total degree $\operatorname{deg}_{f} U_{q}(z, f)=n$ in $f(z)$ and its $q$-shifts, and $\operatorname{deg}_{f} Q_{q}(z, f) \leq n$. Moreover, we assume that $U_{q}(z, f)$ contains just one term of maximal total degree in $f(z)$ and its $q$-shifts. Then

$$
m\left(r, P_{q}(z, f)\right)=o(T(r, f))
$$

on a set of logarithmic density 1.

From Theorems A, B, C and D, we find that $U(z, f)=f(z)^{n}$ or $U(z, f)$ has just one term of maximal total degree $n$ in $f(z)$ and its $(q$-)shifts. It is natural to ask whether the assumption of just one term of maximal total degree $n$ could be removed. This is an open problem raised in [7]. In this paper, we consider difference polynomials $U(z, f)$ and $q$-difference polynomials $U_{q}(z, f)$ which can contain many terms of maximal total degree $n$.

The remainder of the paper is organized as follows. We discuss difference counterparts of the Clunie lemma from the Nevanlinna theory of differential polynomials, where the difference polynomial can contain many terms of maximal total degree in $f(z)$ and its shifts in Section 2. We present $q$-difference counterparts of the Clunie lemma from the Nevanlinna theory of differential polynomials, where the $q$-difference polynomial can contain many terms of maximal total degree in $f(z)$ and its $q$-shifts in Section 3.

\section{Difference counterparts of the Clunie lemma}

THEOREM 2.1. Let $f(z)$ be a transcendental meromorphic solution of finite order $\rho$ of a difference equation of the form

$$
U(z, f) P(z, f)=Q(z, f),
$$

where $U(z, f), P(z, f)$ and $Q(z, f)$ are difference polynomials. The maximal total degree $\operatorname{deg}_{f} U(z, f)=n$ in $f(z)$ and its shifts, the sum of terms of maximal total 
degree in $U(z, f)$ does not vanish identically, and $\operatorname{deg}_{f} Q(z, f) \leq n$. Moreover, we assume that $f(z)$ also satisfies, for each $\varepsilon>0$,

$$
N(r, f)+N\left(r, \frac{1}{f}\right)=O\left(r^{\rho-1+\varepsilon}\right)+S(r, f) .
$$

Then for each $\varepsilon>0$,

$$
m(r, P(z, f))=O\left(r^{\rho-1+\varepsilon}\right)+S(r, f),
$$

possibly outside an exceptional set of finite logarithmic measure.

In order to prove Theorem 2.1 we need the following lemmas.

LEMMA 2.2 [2]. Let $\eta_{1}$ and $\eta_{2}$ be two complex numbers such that $\eta_{1} \neq \eta_{2}$ and let $f(z)$ be a finite-order meromorphic function. Let $\rho$ be the order of $f(z)$; then for each $\varepsilon>0$,

$$
m\left(r, \frac{f\left(z+\eta_{1}\right)}{f\left(z+\eta_{2}\right)}\right)=O\left(r^{\rho-1+\varepsilon}\right) .
$$

LEMMA 2.3 [2]. Let $f(z)$ be a meromorphic function with exponent of convergence of poles $\lambda(r, 1 / f)=\lambda<+\infty, \eta \neq 0$ be fixed. Then for each $\varepsilon>0$,

$$
N(r, f(z+\eta))=N(r, f)+O\left(r^{\lambda-1+\varepsilon}\right)+O(\log r) .
$$

Proof of Theorem 2.1. Set

$$
U(z, f)=\sum_{\nu \in K} c_{\nu}(z) \prod_{j=1}^{v_{v}} f\left(z+\gamma_{\nu, j}\right)^{n_{\nu, j}},
$$

where $K$ is an index set, $\gamma_{v, j} \in \mathbb{C}$, and the coefficients of the difference polynomials (2.4) are small functions as understood in the usual Nevanlinna theory; that is, their characteristic is type $S(r, f)$.

By the assumption in Theorem 2.1, (1.2) and (2.4),

$$
\max _{\mu \in J} \sum_{j=1}^{\tau_{\mu}} m_{\mu, j} \leq n=\max _{\nu \in K} \sum_{j=1}^{v_{\nu}} n_{\nu, j} .
$$

Now we rearrange the expression for the difference polynomial (2.4) by collecting together all terms having the same total degree. Set

$$
\wedge_{k}:=\left\{v \in K \mid \sum_{j=1}^{v_{v}} n_{v, j}=k\right\} .
$$

Then we obtain

$$
\begin{gathered}
U(z, f)=\sum_{\nu \in \wedge_{n}} c_{\nu}(z) \prod_{j=1}^{v_{v}} f\left(z+\gamma_{\nu, j}\right)^{n_{v, j}}+\sum_{\nu \in \wedge_{n-1}} c_{\nu}(z) \prod_{j=1}^{v_{v}} f\left(z+\gamma_{\nu, j}\right)^{n_{\nu, j}}+\cdots \\
+\sum_{\nu \in \wedge_{1}} c_{\nu}(z) \prod_{j=1}^{v_{v}} f\left(z+\gamma_{\nu, j}\right)^{n_{\nu, j}}+\sum_{\nu \in \wedge_{0}} c_{\nu}(z) .
\end{gathered}
$$


Thus we can write $U(z, f)$ in the form

$$
U(z, f)=\widetilde{c}_{n}(z) f(z)^{n}+\widetilde{c}_{n-1}(z) f(z)^{n-1}+\cdots+\widetilde{c}_{1}(z) f(z)+\widetilde{c}_{0}(z),
$$

where

$$
\tilde{c}_{0}(z)=\sum_{\nu \in \wedge_{0}} c_{\nu}(z), \quad \tilde{c}_{k}(z)=\sum_{\nu \in \wedge_{k}} c_{\nu}(z) \prod_{j=1}^{v_{\nu}}\left(\frac{f\left(z+\gamma_{\nu, j}\right)}{f(z)}\right)^{n_{\nu, j}}, \quad j=1, \ldots, n .
$$

We can show that the coefficient $\widetilde{c}_{n}(z)$ in (2.5) does not vanish identically. Suppose that $\widetilde{c}_{n}(z) \equiv 0$; that is,

$$
\tilde{c}_{n}(z)=\sum_{\nu \in \wedge_{n}} c_{\nu}(z) \prod_{j=1}^{v_{\nu}}\left(\frac{f\left(z+\gamma_{\nu, j}\right)}{f(z)}\right)^{n_{\nu, j}} \equiv 0
$$

Then

$$
\begin{aligned}
0 \equiv \widetilde{c}_{n}(z) f(z)^{n} & =\left(\sum_{\nu \in \wedge_{n}} c_{\nu}(z) \prod_{j=1}^{v_{v}}\left(\frac{f\left(z+\gamma_{\nu, j}\right)}{f(z)}\right)^{n_{v, j}}\right) f(z)^{n} \\
& =\sum_{\nu \in \wedge_{n}} c_{\nu}(z) \prod_{j=1}^{v_{v}} f\left(z+\gamma_{v, j}\right)^{n_{v, j}} .
\end{aligned}
$$

This shows that the sum of terms of maximal total degree vanishes identically in $U(z, f)$. This contradicts the assumption in Theorem 2.1.

By Lemma 2.2 and the assumption concerning the coefficients $c_{v}(z)$, we see immediately that

$$
m\left(r, \widetilde{c}_{j}(z)\right)=O\left(r^{\rho-1+\varepsilon}\right)+S(r, f)
$$

for $j=0,1, \ldots, n$.

Moreover, by (2.2) and Lemma 2.3,

$$
N\left(r, \widetilde{c}_{j}(z)\right)=O\left(r^{\rho-1+\varepsilon}\right)+S(r, f), \quad N\left(r, \frac{1}{\widetilde{c}_{j}(z)}\right)=O\left(r^{\rho-1+\varepsilon}\right)+S(r, f),
$$

for $j=0,1, \ldots, n$. Hence

$$
T\left(r, \widetilde{c}_{j}(z)\right)=O\left(r^{\rho-1+\varepsilon}\right)+S(r, f) .
$$

By using the first main theorem of Nevanlinna theory, we have, together with (2.6), (2.7) and (2.8),

$$
m\left(r, \frac{1}{\widetilde{c}_{j}(z)}\right)=O\left(r^{\rho-1+\varepsilon}\right)+S(r, f) .
$$


Now, reasoning as in [8], we define

$$
c(z)=\max _{1 \leq j \leq n}\left\{1,2\left|\frac{\widetilde{c}_{n-j}(z)}{\widetilde{c}_{n}(z)}\right|^{1 / j}\right\} .
$$

Obviously, $c(z)$ is not meromorphic. However, we may compute the proximity function of $c(z)$ and obtain

$$
m(r, c(z)) \leq \sum_{j=0}^{n} m\left(r, \widetilde{c}_{j}(z)\right)+m\left(r, \frac{1}{\widetilde{c}_{n}(z)}\right)+O(1)=O\left(r^{\rho-1+\varepsilon}\right)+S(r, f)
$$

Set

$$
E_{1}=\left\{\theta \in[0,2 \pi):\left|f\left(r e^{i \theta}\right)\right| \leq c\left(r e^{i \theta}\right)\right\}, \quad E_{2}=[0,2 \pi) \backslash E_{1} .
$$

It follows from (1.1), for $z=r e^{i \theta}$ with $\theta \in E_{1}$, that

$$
\begin{aligned}
|P(z, f)| & \leq \sum_{\lambda \in I}\left|a_{\lambda}(z)\right| \prod_{j=1}^{\sigma_{\lambda}}\left|f\left(z+\alpha_{\lambda, j}\right)\right|^{l_{\lambda, j}} \\
& =\sum_{\lambda \in I}\left|a_{\lambda}(z)\right| \cdot|f(z)|^{l_{\lambda}} \prod_{j=1}^{\sigma_{\lambda}}\left|g_{\lambda, j}(z)\right|^{l_{\lambda, j}} \\
& =\sum_{\lambda \in I}\left(\left|a_{\lambda}(z) f(z)^{l_{\lambda}}\right|^{2}\right)^{1 / 2}\left(\prod_{j=1}^{\sigma_{\lambda}}\left|g_{\lambda, j}(z)\right|^{2 l_{\lambda, j}}\right)^{1 / 2} \\
& \leq\left(\sum_{\lambda \in I}\left|a_{\lambda}(z) f(z)^{l_{\lambda}}\right|^{2}\right)^{1 / 2}\left(\sum_{\lambda \in I} \prod_{j=1}^{\sigma_{\lambda}}\left|g_{\lambda, j}(z)\right|^{2 l_{\lambda, j}}\right)^{1 / 2},
\end{aligned}
$$

where $g_{\lambda, j}(z)=f\left(z+\alpha_{\lambda, j}\right) / f(z)$.

On the other hand, for $z=r e^{i \theta}$ with $\theta \in E_{2}$, we have, by (2.9) and (2.11),

$$
|f(z)|>c(z) \geq 2\left|\frac{\widetilde{c}_{n-j}(z)}{\widetilde{c}_{n}(z)}\right|^{1 / j}, \quad|f(z)|>1,
$$

and hence

$$
\left|\frac{\widetilde{c}_{n-j}(z)}{\widetilde{c}_{n}(z)}\right| \leq \frac{|f(z)|^{j}}{2^{j}}, \quad \frac{1}{|f(z)|}<1,
$$

for all $j=1,2, \ldots, n$.

This means that by (2.5) and (2.13), for $z=r e^{i \theta}$ with $\theta \in E_{2}$, we have

$$
|U(z, f)| \geq\left|\widetilde{c}_{n}(z)\right| \cdot|f(z)|^{n}\left(1-\sum_{j=1}^{n}\left|\frac{\widetilde{c}_{n-j}(z)}{\widetilde{c}_{n}(z)}\right| \cdot \frac{1}{|f(z)|^{j}}\right) \geq \frac{\left|\widetilde{c}_{n}(z)\right| \cdot|f(z)|^{n}}{2^{n}}
$$


Now, recalling the assumption on the total degrees of $Q(z, f)$ and $U(z, f)$, by (1.2), (2.1), (2.13) and (2.14), for $z=r e^{i \theta}$ with $\theta \in E_{2}$, we may estimate

$$
\begin{aligned}
|P(z, f)| & =\left|\frac{Q(z, f)}{U(z, f)}\right| \leq \frac{2^{n}}{\left|\widetilde{c}_{n}(z)\right| \cdot|f(z)|^{n}} \sum_{\mu \in J}\left|b_{\mu}(z)\right| \prod_{j=1}^{\tau \mu}\left|f\left(z+\beta_{\mu, j}\right)\right|^{m_{\mu, j}} \\
& \leq \frac{2^{n}}{\left|\widetilde{c}_{n}(z)\right|}\left(\sum_{\mu \in J}\left|b_{\mu}(z)\right|^{2}\right)^{1 / 2}\left(\sum_{\mu \in J} \prod_{j=1}^{\tau_{\mu}}\left|h_{\mu, j}(z)\right|^{2 m_{\mu, j}}\right)^{1 / 2}
\end{aligned}
$$

where $h_{\mu, j}(z)=f\left(z+\beta_{\mu, j}\right) / f(z)$.

Now we estimate

$$
m(r, P(z, f))=\frac{1}{2 \pi} \int_{0}^{2 \pi} \log ^{+}|P(z, f)| d \theta
$$

It follows from (2.12) for the case $\theta \in E_{1}$ and (2.15) for the case $\theta \in E_{2}$, that the last factor on the right-hand side of both of these inequalities yields $O\left(r^{\rho-1+\varepsilon}\right)$ if we take logarithms and integrate them. The factors formed by the coefficients $a_{\lambda}(z)$ and $b_{\mu}(z)$ in (2.12) and (2.15) are of type $S(r, f)$, by the assumption of Theorem 2.1. The factor $\left(2^{n} /\left|\widetilde{c_{n}}(z)\right|\right)$ in the case $\theta \in E_{2}$ results in $O\left(r^{\rho-1+\varepsilon}\right)+S(r, f)$ by (2.10). Thus,

$$
m(r, P(z, f))=\frac{1}{2 \pi} \int_{0}^{2 \pi} \log ^{+}|P(z, f)| d \theta=O\left(r^{\rho-1+\varepsilon}\right)+S(r, f) .
$$

This completes the proof of the theorem.

REMARK 2.4. Theorem 2.1 fails if $U(z, f)$ contains at least two terms of maximal total degree but does not satisfy condition (2.2). Consider the difference equation

$$
\left[f\left(z+\frac{\pi}{2}\right)^{2}+f(z+\pi)^{2}\right] \cdot f(z)=-f(z+\pi)
$$

which is solved by $f(z)=\cos z$, where

$U(z, f)=f\left(z+\frac{\pi}{2}\right)^{2}+f(z+\pi)^{2}, \quad P(z, f)=f(z), \quad Q(z, f)=-f(z+\pi)$.

Obviously, $\operatorname{deg}_{f} U(z, f)=2, \operatorname{deg}_{f} Q(z, f)=1$ and for each $\varepsilon>0$,

$$
N(r, f)+N\left(r, \frac{1}{f}\right) \neq O\left(r^{\rho-1+\varepsilon}\right)+S(r, f) .
$$

We obtain that $m(r, P(z, f))=m(r, \cos z)=2 r / \pi$ does not satisfy (2.3). 


\section{3. $q$-Difference counterparts of the Clunie lemma}

THEOREM 3.1. Let $f(z)$ be a transcendental meromorphic solution of zero order of a q-difference equation of the form

$$
U_{q}(z, f) P_{q}(z, f)=Q_{q}(z, f),
$$

where $U_{q}(z, f), P_{q}(z, f)$ and $Q_{q}(z, f)$ are $q$-difference polynomials. The total degree $\operatorname{deg}_{f} U_{q}(z, f)=n$ in $f(z)$ and its $q$-shifts, the sum of terms of maximal total degree in $U_{q}(z, f)$ does not vanish identically, and $\operatorname{deg}_{f} Q_{q}(z, f) \leq n$. Moreover, we assume that $f(z)$ also satisfies

$$
N(r, f)+N\left(r, \frac{1}{f}\right)=o(T(r, f)) .
$$

Then

$$
m\left(r, P_{q}(z, f)\right)=o(T(r, f))
$$

on a set of logarithmic density 1.

In order to prove the Theorem 3.1, we first state a lemma.

LEMMA 3.2 [2]. Let $f(z)$ be a nonconstant zero-order meromorphic function, and $q \in \mathbb{C} \backslash\{0,1\}$. Then

$$
m\left(r, \frac{f(q z)}{f(z)}\right)=o(T(r, f))
$$

on a set of logarithmic density 1.

Proof OF THEOREM 3.1. The proof is completely parallel to the proof of Theorem 2.1. We again rearrange the expression for the $q$-difference polynomial $U_{q}(z, f)$ in the form

$$
U_{q}(z, f)=\tilde{\gamma}_{n}(z) f(z)^{n}+\tilde{\gamma}_{n-1}(z) f(z)^{n-1}+\cdots+\tilde{\gamma}_{1}(z) f(z)+\tilde{\gamma}_{0}(z),
$$

where each coefficient $\tilde{\gamma}_{j}(z), j=0,1, \ldots, n$, is the sum of finitely many terms of the form $f\left(q^{k} z\right) / f(z)$, each such product being multiplied by one of the original coefficients of $U_{q}(z, f)$. By using similar reasoning, as applied to $\widetilde{c}_{n}(z)$ in Section 2, we assert that $\widetilde{\gamma}_{n}(z)$ does not vanish identically.

Thus we obtain, by Lemma 3.2 and the assumption on the original coefficients of $U_{q}(z, f)$,

$$
m\left(r, \tilde{\gamma}_{j}(z)\right)=o(T(r, f)),
$$

for all $j=0,1, \ldots, n$, on a set of logarithmic density 1 . Moreover, by (3.1),

$$
N\left(r, \tilde{\gamma}_{j}(z)\right)=o(T(r, f)), \quad N\left(r, \frac{1}{\widetilde{\gamma}_{j}(z)}\right)=o(T(r, f)),
$$


for all $j=0,1, \ldots, n$, on a set of logarithmic density 1 . Hence

$$
T\left(r, \tilde{\gamma}_{j}(z)\right)=o(T(r, f))
$$

for all $j=0,1, \ldots, n$, on a set of logarithmic density 1 .

Now using the first main theorem of Nevanlinna theory, we have, by (3.2), (3.3) and (3.4),

$$
m\left(r, \frac{1}{\widetilde{\gamma}_{j}(z)}\right)=o(T(r, f)),
$$

for all $j=0,1, \ldots, n$, on a set of logarithmic density 1 . Defining as before,

$$
\gamma(z)=\max _{1 \leq j \leq n}\left\{1,2\left|\frac{\tilde{\gamma}_{n-j}(z)}{\widetilde{\gamma}_{n}(z)}\right|^{1 / j}\right\} .
$$

Although $\gamma(z)$ is not meromorphic, we may compute the proximity function of $\gamma(z)$ and obtain

$$
m(r, \gamma(z))=o(T(r, f))
$$

on a set of logarithmic density 1 .

Set

$$
F_{1}=\left\{\theta \in[0,2 \pi):\left|f\left(r e^{i \theta}\right)\right| \leq \gamma\left(r e^{i \theta}\right)\right\}, \quad F_{2}=[0,2 \pi) \backslash F_{1} .
$$

We may now complete the proof by reasoning as in the proof of Theorem 2.1.

\section{Acknowledgement}

The authors are very grateful to the referee for many valuable comments and suggestions which greatly improved the presentation of this paper.

\section{References}

[1] D. C. Barnett, R. G. Halburd, R. J. K. Orhonen and W. Morgan, 'Nevanlinna theory for the $q$-difference operator and meromorphic solutions of $q$-difference equations', Proc. Roy. Soc. Edinburgh Sect. A 137 (2007), 457-474.

[2] Y. M. Chiang and S. J. Feng, 'On the Nevanlinna characteristic of $f(z+\eta)$ and difference equations in the complex plane', Ramanujan J. 16 (2008), 105-129.

[3] J. Clunie, 'On integral and meromorphic functions', J. London Math. Soc. 37 (1962), 17-27.

[4] R. G. Halburd and R. J. Korhonen, 'Difference analogue of the lemma on the logarithmic derivative with applications to difference equations', J. Math. Anal. Appl. 314 (2006), 477-487.

[5] Y. He and X. Xiao, Algebroid Functions and Ordinary Differential Equations (Science Press, Beijing, 1988).

[6] I. Laine, Nevanlinna Theory and Complex Differential Equations (Walter de Gruyter, Berlin, 1993). 
[7] I. Laine and C. C. Yang, 'Clunie theorems for difference and $q$-difference polynomials', J. London Math. Soc. 76 (2007), 556-566.

[8] C. C. Yang and Z. Ye, 'Estimates of the proximate function of differential polynomials', Proc. Japan Acad. Ser. A Math. Sci. 83 (2007), 50-55.

ZHI-BO HUANG, School of Mathematical Sciences, South China Normal University, Guangzhou 510631, PR China

e-mail: hzbo20019@sina.com

ZONG-XUAN CHEN, School of Mathematical Sciences,

South China Normal University, Guangzhou 510631, PR China

e-mail: chzx@vip.sina.com 